\title{
Association and interaction analysis of PPARGC1A and serum uric acid on type 2 diabetes mellitus in Chinese Han population
}

Hui-Hui Wu', Nai-Jia Liu', Zhen Yang ${ }^{3}$, Xiao-Ming Tao ${ }^{4}$, Yan-Ping Du' ${ }^{4}$, Xuan-Chun Wang ${ }^{1}$, Bin Lu', Zhao-Yun Zhang ${ }^{1}$, Ren-Ming Hu${ }^{1}$ and Jie Wen ${ }^{1,2^{*}}$

\begin{abstract}
Background: Peroxisome proliferator-activated receptor gamma coactivator-1a (PPARGC1A/ PGC-1a) is a ligand-activated transcription factor belonging to the nuclear hormone receptor superfamily. The activity of PGC-1a or genetic variations in the gene encoding the enzyme may contribute to individual variations in mitochondrial function and insulin resistance or diabetes. The objective of this study was to assess the extent to which PPARGC1A (rs8192678) and serum uric acid (UA) and its interaction impact on T2DM susceptibility in Chinese Han population.

Method: We conducted a study in a cohort that included 1166 T2DM patients and 1135 controls, and was genotyped for the presence of the PPARGC1A rs8192678 polymorphisms. Genotyping was performed by iPLEX technology. The association between rs 8192678 or UA and T2DM was assessed by univariate and multivariate logistic regression (MLR) analysis controlling for confounders. The interaction between rs8192678 and UA for T2DM susceptibility was also assessed by MLR analysis.

Results: The generalized linear regression analysis failed to show an association between the PPARGC1A rs8192678 polymorphisms and T2DM. Interestingly, the present study provided data suggesting that the minor A-allele of PPARGC1A (rs8192678) had a protective effect against T2DM in subjects with higher level of UA (OR int $=1.5095 \%$ Cl: 1.06-2.12 for allele and $P=0.02, \mathrm{OR}_{\text {int }}=1.6395 \% \mathrm{Cl}: 1.17-2.26$ for genotype and $\mathrm{P}=0.004$ ).

Conclusion: The combination of higher level of UA and PPARGC1A (rs8192678) was an independent predictor for T2DM.
\end{abstract}

Keyword: PPARGC1A, Serum uric acid, Interaction, Type 2 diabetes mellitus, Chinese Han population

\section{Introduction}

Type 2 diabetes mellitus (T2DM) is a complex metabolic disorder characterized by hyperglycemia as a result of pancreatic beta cell dysfunction and insulin resistance [1]. There is evidence demonstrating that genetic determinants as well as environmental factors may play a role in the pathophysiology of T2DM. Identifying rare and common genetic variants contributing to risk of or protection from T2DM will help uncover the complex mechanisms

\footnotetext{
*Correspondence: wenjie065@126.com

'Department of Endocrinology and Metabolism, Huashan Hospital, Fudan University, NO. 12 Wulumuqi Mid Road, Building 0\#, Jing'an District, Shanghai 200040, China

${ }^{2}$ Department of Endocrinology and Metabolism, Jing'an District Center Hospital of Shanghai, Shanghai 200040, China

Full list of author information is available at the end of the article
}

underlying T2DM, and provide crucial implications for the development of personalized medicine for diabetes mellitus [2].

Peroxisome proliferator-activated receptor gamma coactivator-1 $\alpha$ (PPARGC1A/PGC- $1 \alpha$ ) is a ligand-activated transcription factor belonging to the nuclear hormone receptor superfamily, named after its ability to bind chemicals known to induce peroxisome proliferation [3]. Numerous studies have identified an important role for PPARGC1A in gluconeogenesis and insulin sensitivity as well as the beta-oxidation of fatty acids in the liver [4]. Plasma fasting insulin has been linked to the chromosomal region where the PPARGC1A gene is located [5], which verify the hypothesis that the gene may be a functional and positional candidate for T2DM. Moreover, 
endothelial PPARGC1A has been found to repress endothelial migration, thus potently inhibit endothelial function and angiogenesis, which further contribute to multiple aspects of vascular dysfunction in diabetes [6]. Associations of PPARGC1A variants with a range of other metabolic traits, including glucose concentrations, dyslipidemia and obesity have been reported [7-10]. The PPARGC1A rs8192678 polymorphism encodes a missense amino acid change, however, the activity of PGC- $1 \alpha$ or genetic variations in the gene may contribute to individual variations in mitochondrial function and insulin resistance or diabetes $[11,12]$. Recently, a new window has opened on the possible associations of PPARGC1A (rs8192678) with several metabolic related traits [13]. Furthermore, PPARGC1A (rs8192678) has also been found to be associated with a higher risk of T2DM in Indian and East Asian populations [14,15]. Uncovering the effect of PPARGC1A variant on the susceptibility of T2DM in Chinese population and how it functions within the regulatory network will deepen our understanding of the biological roles of PPARGC1A.

In the relevant network, cellular PPARGC1A is regulated by signaling inputs that increase the transcription of the PPARGC1A gene and activity of PPARGC1A protein [16]. Emerging evidence has been provided to illustrate that PPARGC1A transcription increases with exercise, cold exposure, fasting and electro acupuncture [17-19]. It is thereby feasible that environment stimuli which could irritate the transcription of PPARGC1A gene also participate in modulation of T2DM susceptibility.

Serum uric acid (UA), as the final oxidation product of purine catabolism, has been associated with various clinical conditions, such as diabetes mellitus (DM), atherosclerotic disease and abdominal obesity [20-23]. Significant interactions between UA and age, triglycerides, as well as metabolic syndrome have also been reported in numerous studies [24-26]. There are few studies available on the effect of the interaction between UA and genetic variants on diseases. The objective of this study was to clarify whether the rs8192678 polymorphism or UA is associated with T2DM in Chinese population and to determine whether rs8192678 interacts with UA to impact on T2DM risk.

\section{Materials and methods Study population}

The study protocol was approved by the Ethics Committee of Huashan Hospital, Shanghai, China. We carried out a study in a random sample of Chinese individuals to evaluate the association between PPARGC1A (rs8192678) and T2DM, as well as to determine whether the variant interacts with UA to influence T2DM susceptibility. Participants enrolled in the cohort, including 1166 T2DM patients and 1135 controls, were of Southern Han Chinese ancestry residing in the Shanghai metropolitan area. T2DM patients registered in the analysis were recruited from the Endocrinology and Metabolism outpatient clinics at Fudan University Huashan Hospital in Shanghai. Written consent was obtained from all patients before the study.

\section{Measurement}

The subjects were interviewed for the documentation of medical histories, medications and regular physical examinations. Systolic and diastolic blood pressure (BP) values were the means of two physician-obtained measurements on the left arm of the seated participant. Body mass index (BMI) was calculated as the weight in kilograms divided by the square of height in meters. All participants underwent a complete hematological examination in the fasting state. Plasma glucose was quantified by the glucose oxidase-peroxidase procedure. Serum total cholesterol (TC), triglyceride (TG), high-density lipoprotein (HDL) cholesterol, urea nitrogen (UN), uric acid (UA) and alanine transaminase (ALT) levels were measured by an enzymatic method with a chemical analyzer (Hitachi 7600-020, Tokyo, Japan). Low-density lipoprotein (LDL) cholesterol levels were calculated using the Friedewald formula. The day-to-day and inter-assay coefficients of variation at the central laboratory in our hospital for all analyses were between $1 \%$ and $3 \%$.

\section{Definition}

Hypertension (HT) was defined as blood pressure $\geq 140$ / $90 \mathrm{mmHg}$ or history of hypertension medication. The classification of serum uric acid (UA) was based on the Chinese criteria [24]: UA $\geq 420 \mathrm{umol} / \mathrm{L}$. Diabetes was defined according to $1999 \mathrm{WHO}$ criteria [27]: fasting plasma glucose $\geq 7.0 \mathrm{mmol} / \mathrm{L}$, and/or 2-hour plasma glucose $\geq 11.1 \mathrm{mmol} / \mathrm{L}$ in oral glucose tolerance test (OGTT). Known subtypes of diabetes were excluded based on antibody measurements and inheritance. The non-diabetic unrelated individuals meeting the following criterions were identified as the control population: 1) no family history of diabetes; 2) $\geq 45$ years of age; 3 ) normal glucose tolerance verified by OGTT. The clinical characteristics of participants are summarized in Table 1.

\section{SNP genotyping}

Peripheral venous blood samples were collected from all study subjects, and the genomic DNA was isolated from whole blood by proteinase $\mathrm{K}$ digestion followed by phenol-chloroform extraction. PPARGC1A (rs8192678) was genotyped using iPLEX (Sequenom, San Diego, CA, USA) and detected by matrix-assisted laser desorption/ ionization-time of flight mass spectrometry in a total of 2301 Chinese Han individuals. The genotype frequency was in Hardy-Weinberg equilibrium $(P>0.05)$ and there 
Table 1 The clinical characteristics of subjects

\begin{tabular}{lll}
\hline Variable & Diabetes & Control \\
\hline $\mathrm{N}$ & 1166 & 1135 \\
Age & $65.46 \pm 10.56$ & $59.09 \pm 7.85$ \\
Sex (male/female) & $456 / 710$ & $352 / 783$ \\
Height & $160.2 \pm 8.64$ & $161.09 \pm 7.65$ \\
Weight & $64.85 \pm 10.71$ & $62.78 \pm 10.15$ \\
SBP & $139.52 \pm 19.92$ & $126.44 \pm 16.92$ \\
DBP & $82.88 \pm 10.98$ & $80.52 \pm 10.13$ \\
UN & $6.08 \pm 1.63$ & $5.62 \pm 1.37$ \\
UA & $0.30 \pm 0.08$ & $0.31 \pm 0.08$ \\
FPG & $8.39 \pm 3.03$ & $5.22 \pm 0.38$ \\
PPG & $15.05 \pm 5.34$ & $6.03 \pm 1.04$ \\
TC & $5.43 \pm 1.11$ & $5.35 \pm 1.00$ \\
TG & $2.00 \pm 1.46$ & $1.47 \pm 1.06$ \\
HDL & $1.29 \pm 0.34$ & $1.43 \pm 0.36$ \\
LDL & $3.11 \pm 0.86$ & $3.10 \pm 0.79$ \\
ALT & $28.44 \pm 16.08$ & $24.04 \pm 13.69$ \\
rS8192678 (A/G) & $490 / 670$ & $475 / 646$ \\
\hline
\end{tabular}

Note: SBP-systolic blood pressure, DBP-diastolic blood pressure, UN-urea nitrogen, UA-uric acid, FPG-fasting plasma glucose, PBG-postprandial blood glucose, TC-total cholesterol, TG-triglyceride, HDL-high density lipoprotein, LDL-low density lipoprotein, ALT-alanine transaminase.

was a $99.9 \%$ genotype concordance rate when duplicated samples were compared across plates.

\section{Statistical analysis}

The Kolmogorov-Smirnov test was used to determine whether continuous variables followed a normal distribution. Variables that were not normally distributed were log-transformed to approximate normal distribution for analysis. Results are described as mean \pm SD or median unless stated otherwise. Differences in variables between T2DM and control were determined by unpaired $t$-test. Between groups differences in properties were accessed by $\chi^{2}$ analysis. Univariate logistic regression was performed to determine variables associated with T2DM and to estimate confounding factors possibly disturbing the relation of UA and/or PPARGC1A to T2DM. Multivariable logistic regression (MLR) was carried out to control potential confounders for determining independent contribution of variables to T2DM. For interaction analysis, MLR was conducted to include two main variables and its interaction item to determine the interaction effect. In order to better investigate the interaction between UA and PPARGC1A on T2DM, we performed two analyses according to alleles and genotypes of PPARGC1A that were present in the study population by additive model. Odds ratios (OR) with 95\% confidence intervals (CI) were calculated as measures of association of UA and/or PPARGC1A with T2DM. Results were analyzed using the Statistical
Table 2 Univariate logistic regression analysis for diabetes

\begin{tabular}{llllll}
\hline Variable & $\boldsymbol{\beta}$ & S.E. & P Value & OR & $\mathbf{9 5 \% ~ C l}$ \\
\hline Age & 0.07 & 0.003 & $<0.001$ & 1.07 & $1.07-1.08$ \\
Sex & -0.35 & 0.06 & $<0.001$ & 0.70 & $0.62-0.80$ \\
BMl & 0.59 & 0.08 & $<0.001$ & 1.81 & $1.54-2.14$ \\
HT & 1.21 & 0.09 & $<0.001$ & 3.36 & $2.83-3.99$ \\
TC & 0.07 & 0.03 & 0.01 & 1.07 & $1.02-1.14$ \\
TG & 0.46 & 0.03 & $<0.001$ & 1.59 & $1.48-1.70$ \\
UN & 0.21 & 0.02 & $<0.001$ & 1.24 & $1.19-1.29$ \\
UA & 0.03 & 0.09 & 0.74 & 1.03 & $0.87-1.22$ \\
PPARGC1A & -0.005 & 0.06 & 0.93 & 0.99 & $0.88-1.12$ \\
\hline
\end{tabular}

Note: PPARGC1A with SNP (rs8192678), BMI- body mass index, TC-total cholesterol, TG-triglyceride, UN-urea nitrogen, UA-uric acid, HT-Hypertension.

Package for Social Sciences for Windows version 16.0 (SPSS, Chicago, IL, USA). Tests were two-sided and a p-value of $<0.05$ was considered significant.

\section{Results}

\section{Clinical characteristics of subjects}

The baseline clinical characteristics of the 2301 subjects were listed in Table 1. There are 456 males and 710 females (mean age, $65.46 \pm 10.56$ years) in cases and 352 males and 783 females (mean age, $59.09 \pm 7.85$ years) in controls. Diabetic patients had more weight than controls. SBP, DBP, FPG, PPG, TC and TG levels were higher in cases than those of controls, while HDL level was lower in cases. Serum UA, ALT and LDL levels were similar between the two groups. The minor allele (A) frequency of PPARGC1A was $42.24 \%$ and $42.37 \%$ in cases and controls, respectively.

\section{Univariate and multiple logistic regression analysis for diabetes}

To estimate the association of various clinical factors and T2DM, univariate logistic regression models were developed to include age, sex, hypertension, lipid profiles, UA and the rs 8192678 polymorphisms (Table 2). The univariate logistic analyses indicated that age, sex, BMI, hypertension and TC were significantly associated with T2DM $(\mathrm{P}<0.05$ for all), however, there were no significant association of UA and SNP (rs8192678) with T2DM ( $\mathrm{P}=0.744$ and 0.928 , respectively). The proportion of T2DM was $52.17 \%$ and $51.75 \%$ in low UA group and high UA group,

Table 3 The interaction effect analysis of UA and allele of PPARGC1A (rs8192678) for diabetes

\begin{tabular}{lccrcr}
\hline Variable & $\boldsymbol{\beta}$ & S.E. & $\boldsymbol{P}$ Value & OR & $\mathbf{9 5 . 0 \%}$ C.I \\
\hline UA & -0.12 & 0.11 & 0.28 & 0.89 & $0.71-1.10$ \\
rs8192678(A/G) & -0.06 & 0.06 & 0.32 & 0.94 & $0.83-1.06$ \\
UA by rs8192678(A/G) & 0.41 & 0.18 & 0.02 & 1.50 & $1.06-2.12$
\end{tabular}

Note: Variable - UA, PPARGC1A (rs8192678), UA by PPARGC1A (rs8192678); Adjusted for Age, Sex, HT, BMI. 


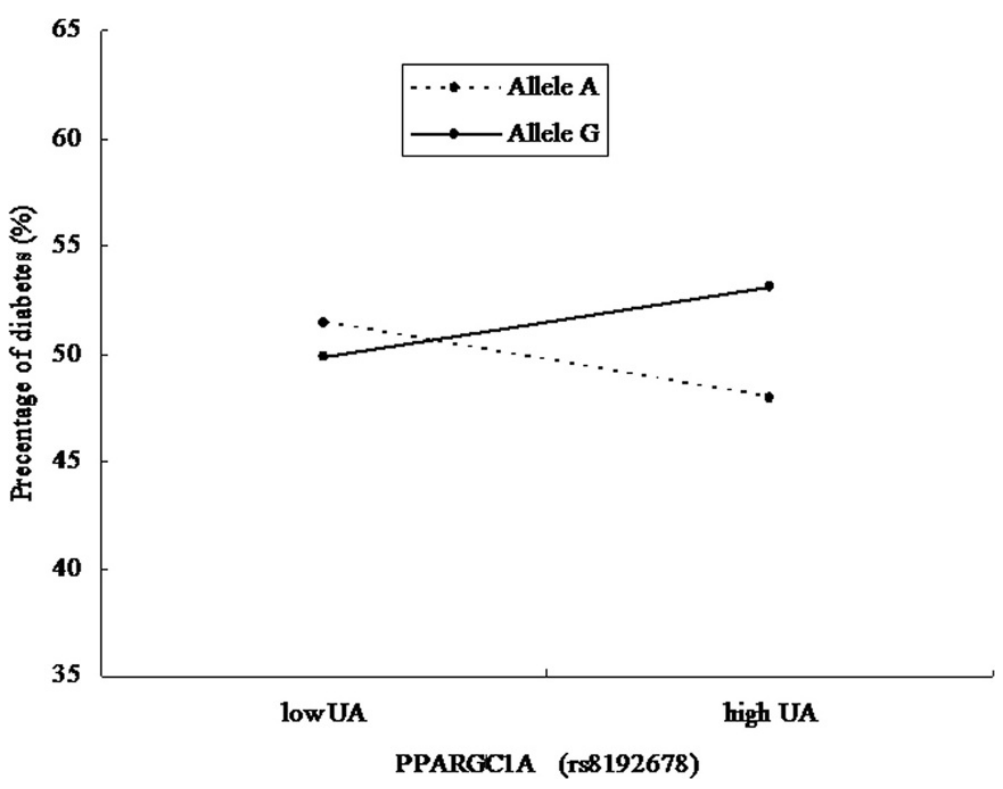

Figure 1 The interaction effect analysis of UA and PPARGC1A for diabetes.

respectively, but the difference was not statistically significant between the two groups $(\mathrm{P}=0.744)$.

\section{UA by PPARGC1A interaction analysis for diabetes}

The interaction item between UA and PPARGC1A, as well as the interaction effect were detected in MLR models after adjustment for relevant potential confounders, showing a certain relationship between them $\left(\mathrm{P}=0.02, \mathrm{OR}_{\mathrm{Int}}=\right.$ 1.50, 95\% CI 1.06-2.12, shown in Table 3 and Figure 1). In model of rs 8192678 with allele variable, MLR models were developed to include the two main effect variables: UA and rs8192678. In another model of rs8192678 with genotype variable, the interaction term of UA and rs8192678 was detected by the same method ( $\mathrm{P}=0.004$, Table 4$)$, as well as the interaction effect $\left(\mathrm{OR}_{\mathrm{Int}}=1.625,95 \% \mathrm{CI} 1.172\right.$ 2.255). In these two models, MLR models signified that UA or rs8192678 alone was not significantly associated with T2DM. The interaction term of UA and SNP rs8192678 which was detected in the two models (allele and genotype models) only confirmed a significant association between this SNP and T2DM in high UA group ( $\mathrm{P}=$ 0.018 for allele analysis and $\mathrm{P}<0.01$ for genotype analysis, data not shown).

Table 4 The interaction effect analysis of UA and genotype of PPARGC1A (rs8192678) for diabetes

\begin{tabular}{lrrrrr}
\hline Variable & $\boldsymbol{\beta}$ & $\boldsymbol{S}$ S.E. & $\boldsymbol{P}$ Value & OR & $\mathbf{9 5 . 0 \%}$ C.I \\
\hline UA & -0.19 & 0.19 & 0.33 & 0.83 & $0.57-1.20$ \\
rs8192678(AA/AG/GG) & -0.04 & 0.07 & 0.60 & 0.96 & $0.84-1.10$ \\
UA by rs8192678 & 0.49 & 0.17 & 0.004 & 1.63 & $1.17-2.26$ \\
\hline
\end{tabular}

Note: Variable - UA, PPARGC1A (rs8192678), UA by PPARGC1A (rs8192678); Adjusted for Age, Sex, HT, BMI.

\section{Discussion}

Our current study evaluated the association between PPARGC1A and T2DM in a case-control trial, subjects enrolled in which were of Chinese Han population including 1166 T2DM patients and 1135 controls. Genotyping was performed for PPARGC1A (rs8192678) in the cohort. This is the first report to our knowledge of an interaction analysis on T2DM susceptibility based on variables of UA and PPARGC1A.

Defect in mitochondrial oxidative phosphorylation have been linked to insulin resistance [28], and there is also evidence suggesting that polymorphisms in PPARGC1A are associated with an increased relative risk of type 2 diabetes, defects in insulin secretion, and lipid oxidation [29]. PPARGC1A is a transcriptional regulator of genes responsible for mitochondrial biogenesis and fat oxidation. Consistently, PPARGC1A (rs8192678) was reported to be associated with T2DM in a European population, as well as diabetic nephropathy (DN) in an Asian Indian population [30,31]. These additional findings, which so far have been observed in numerous studies, showed that PPARGC1A may be a potential genetic marker for T2DM. In our present study, the interaction of rs8192678 (G allele) and high concentration of UA conferred a high risk of T2DM (OR in Tables 3 and 4, Figure 1), while a combination of the A-allele and high UA concentration seemed to protect against T2DM. However, there is little evidence to demonstrate PPARGC1A (rs8192678) to be an independent risk factor of T2DM. Such results may be attributed to the limited number of participants which had insufficient statistical power to detect a slight effect of the common polymorphism in PPARGC1A on T2DM 
susceptibility. A larger sample size, therefore, is necessary to detect the association between this PPARGC1A genetic variant and T2DM. In addition, another possibility of this puzzling phenomenon is the diverse ethnic/regional backgrounds, that is, findings might vary by population because of the underlying unobserved genetic variation.

As mentioned above, the PPARGC1A gene, transcription of which increases with environment stimuli, is a master regulator of mitochondrial genes. Interestingly, recently studies showed an improved intrinsic mitochondrial function in PPARGC1A-overexpressing mice, but only when fatty acids are used as a substrate [32]. Associations between PPARGC1A genotypes and alcohol consumption have been observed in a Mediterranean population [33]. The persuasive arguments confirm the hypothesis that intake of alcohol and fatty acid infusion/supplementation may influence T2DM risk through their modulation in PPARGC1A transcription. On this basis, we suspected that UA may also modify the transcription of PPARGC1A which further regulate the morbidity of diabetes mellitus. Our study is the first analysis to detect the interaction effect of UA and PPARGC1A (rs8192678) on T2DM susceptibility. It is noteworthy that the risk imparted by the wild G-allele was statistically significantly higher than that of A-allele in subjects with higher levels of UA ( $<<0.05$ for allele analysis and $\mathrm{P}<0.01$ for genotype analysis), despite the lack of a direct link between UA level and T2DM. The amusing results prompted the speculation of possible interaction between PPARGC1A (rs8192678) and UA level in determining overall T2DM risk. However, the underlying mechanism is yet to be determined.

Several limitations of the study warrant comment. First, subjects participated in the study were recruited from Shanghai, so they may not have been representative of China as a whole. Second, since our study was performed on Chinese individuals, these findings may not be relevant to people of other ethnicities. Also, it is important to confirm the verdict with additional investigations using a larger sample to systematically evaluate the likely interaction effect of PPARGC1A variants and UA on T2DM risk.

In conclusion, the possible association of PPARGC1A (rs8192678) with T2DM has not been confirmed in the present study. Interestingly, the data also suggested that the minor A-allele of PPARGC1A (rs8192678) had a protective effect against T2DM in subjects with higher levels of UA. However, a functional study, such as gene-targeting in mice, is needed to clarify the role of PPARGC1A as a whole.

\section{Competing interests}

The authors declare that they have no competing interests.

\section{Authors' contributions}

$\mathrm{H}-\mathrm{HW}$ analyzed data and wrote the manuscript. N-JL, ZY, X-MT, Y-PD, X-CW, $\mathrm{BL}$ and $\mathrm{Z}-\mathrm{YZ}$ contributed samples, reagents and analysis tools. JW and $\mathrm{R}-\mathrm{MH}$ conceived and designed the study. All authors read and approved the final manuscript.

\section{Acknowledgements}

We thank the grants from National Natural Science Foundation of China (No. 81270903,30900501, 81370884) and Natural Science Foundation of Shanghai City (No.10ZR1405500) to support the study.

\section{Author details}

${ }^{1}$ Department of Endocrinology and Metabolism, Huashan Hospital, Fudan University, NO. 12 Wulumuqi Mid Road, Building 0\#, Jing'an District, Shanghai 200040, China. ²Department of Endocrinology and Metabolism, Jing'an District Center Hospital of Shanghai, Shanghai 200040, China. ${ }^{3}$ Department of Endocrinology and Metabolism, Xin Hua Hospital, Shanghai Jiao Tong University, Shanghai 200020, China. ${ }^{4}$ Department of Endocrinology and Metabolism, Hua Dong Hospital, Fudan University, Shanghai 200040, China.

Received: 12 May 2014 Accepted: 26 September 2014 Published: 1 October 2014

\section{References}

1. Ndisang JF, Rastogi S, Vannacci A: Insulin resistance, type 1 and type 2 diabetes, and related complications: current status and future perspective. J Diabetes Res 2014, 2014:276475.

2. Groop L, Pociot F: Genetics of diabetes-are we missing the genes or the disease? Mol Cell Endocrinol 2014, 382(1):726-739.

3. Feige JN, Gelman L, Michalik L, Desvergne B, Wahli W: From molecular action to physiological outputs: peroxisome proliferator-activated receptors are nuclear receptors at the crossroads of key cellular functions. Prog Lipid Res 2006, 45(2):120-159.

4. Yoon JC, Puigserver P, Chen G, Donovan J, Wu Z, Rhee J, Adelmant G, Stafford J, Kahn CR, Granner DK, Newgard CB, Spiegelman BM: Control of hepatic gluconeogenesis through the transcriptional coactivator PGC-1. Nature 2001, 413(6852):131-138.

5. Pratley RE, Thompson DB, Prochazka M, Baier L, Mott D, Ravussin E, Sakul H, Ehm MG, Burns DK, Foroud T, Garvey WT, Hanson RL, Knowler WC, Bennett PH, Bogardus C: An autosomal genomic scan for loci linked to prediabetic phenotypes in Pima Indians. J Clin Invest 1998, 101(8):1757-1764.

6. Sawada N, Jiang A, Takizawa F, Safdar A, Manika A, Tesmenitsky Y, Kang KT, Bischoff J, Kalwa H, Sartoretto JL, Kamei Y, Benjamin LE, Watada H, Ogawa Y, Higashikuni Y, Kessinger CW, Jaffer FA, Michel T, Sata M, Croce K, Tanaka R, Arany Z: Endothelial PGC-1alpha mediates vascular dysfunction in diabetes. Cell Metab 2014, 19(2):246-258.

7. Jablonski KA, McAteer JB, de Bakker PI, Franks PW, Pollin TI, Hanson RL, Saxena R, Fowler S, Shuldiner AR, Knowler WC, Altshuler D, Florez JC, Diabetes Prevention Program Research Group: Common variants in 40 genes assessed for diabetes incidence and response to metformin and lifestyle intervention in the diabetes prevention program. Diabetes 2010, 59(10):2672-2681.

8. Povel CM, Feskens EJ, Imholz S, Blaak EE, Boer JM, Dollé ME: Glucose levels and genetic variants across transcriptional pathways: interaction effects with BMI. Int J Obes (Lond) 2010, 34(5):840-845.

9. Franks PW, Ekelund U, Brage S, Luan J, Schafer AJ, O'Rahilly S, Barroso I, Wareham NJ: PPARGC1A coding variation may initiate impaired NEFA clearance during glucose challenge. Diabetologia 2007, 50(3):569-573.

10. Edwards TL, Velez Edwards DR, Villegas R, Cohen SS, Buchowski MS, Fowke JH, Schlundt D, Long J, Cai Q, Zheng W, Shu XO, Hargreaves MK, Smith J, Williams SM, Signorello LB, Blot WJ, Matthews CE: HTR1B, ADIPOR1, PPARGC1A, and CYP19A1 and obesity in a cohort of Caucasians and African Americans: an evaluation of gene-environment interactions and candidate genes. Am J Epidemiol 2012, 175(1):11-21.

11. Choi YS, Hong JM, Lim S, Ko KS, Pak YK: Impaired coactivator activity of the Gly482 variant of peroxisome proliferator-activated receptor gamma coactivator-1alpha (PGC-1alpha) on mitochondrial transcription factor A (Tfam) promoter. Biochem Biophys Res Commun 2006, 344(3):708-712.

12. Ling C, Poulsen $P$, Carlsson E, Ridderstråle M, Almgren P, Wojtaszewski J, Beck-Nielsen H, Groop L, Vaag A: Multiple environmental and genetic factors influence skeletal muscle PGC-1alpha and PGC-1beta gene expression in twins. J Clin Invest 2004, 114(10):1518-1526.

13. Albuquerque $D$, Nóbrega $C$, Rodríguez-López R, Manco L: Association study of common polymorphisms in MSRA, TFAP2B, MC4R, NRXN3, PPARGC1A, TMEM18, SEC16B, HOXB5 and OLFM4 genes with obesity-related traits among Portuguese children. J Hum Genet 2014, 59(6):307-313. 
14. Yang $Y$, Mo X, Chen S, Lu X, Gu D: Association of peroxisome proliferatoractivated receptor gamma coactivator 1 alpha (PPARGC1A) gene polymorphisms and type 2 diabetes mellitus: a meta-analysis. Diabetes Metab Res Rev 2011, 27(2):177-184.

15. Ek J, Andersen G, Urhammer SA, Gaede PH, Drivsholm T, Borch-Johnsen K, Hansen T, Pedersen $\mathrm{O}$ : Mutation analysis of peroxisome proliferatoractivated receptor-gamma coactivator-1 (PGC-1) and relationships of identified amino acid polymorphisms to Type II diabetes mellitus. Diabetologia 2001, 44(12):2220-2226.

16. Collins S, Yehuda-Shnaidman E, Wang H: Positive and negative control of Ucp1 gene transcription and the role of beta-adrenergic signaling networks. Int J Obes (Lond) 2010, 34(Suppl 1):S28-S33.

17. Du H, Zhou C, Wu H, Shan T, Wu Z, Xu B, Zhang Y: Effects of electroacupuncture on PGC-1 alpha expression in brown adipose tissue. Evid Based Complement Alternat Med 2013, 2013:625104.

18. Olesen J, Kiilerich K, Pilegaard H: PGC-1alpha-mediated adaptations in skeletal muscle. Pflugers Arch 2010, 460(1):153-162.

19. Wang L, Liu J, Saha P, Huang J, Chan L, Spiegelman B, Moore DD: The orphan nuclear receptor SHP regulates PGC-1alpha expression and energy production in brown adipocytes. Cell Metab 2005, 2(4):227-238.

20. Yin X, Zhou J, Yu D, Pan Q, Dong X, Zheng F, Li H: The correlation between serum uric acid level and abdominal obesity or metabolic syndrome. Zhonghua Nei Ke Za Zhi 2014, 53(1):13-18.

21. Rafieian-Kopaei M, Behradmanesh $\mathrm{S}$, Kheiri $\mathrm{S}$, Nasri H: Association of serum uric Acid with level of blood pressure in type 2 diabetic patients. Iran $\mathrm{J}$ Kidney Dis 2014, 8(2):152-154.

22. Causevic A, Semiz S, Macic Dzankovic A, Cico B, Dujic T, Malenica M, Bego T: Relevance of uric Acid in progression of type 2 diabetes mellitus. Bosn $J$ Basic Med Sci 2010, 10(1):54-59.

23. Perticone F, Maio R, Tassone JE, Perticone M, Pascale A, Sciacqua A, Sesti G: Interaction between uric acid and endothelial dysfunction predicts new onset of diabetes in hypertensive patients. Int J Cardiol 2013, 167(1):232-236.

24. Tang ZH, Fang Z, Zeng F, Li Z, Zhou L: Association and interaction analysis of metabolic syndrome and serum uric acid on diastolic heart failure. J Endocrinol Invest 2013, 36(8):579-583.

25. Kawamoto R, Tabara Y, Kohara K, Kusunoki T, Abe M, Miki T: Interaction between serum uric acid and triglycerides in relation to prehypertension in community-dwelling Japanese adults. Clin Exp Hypertens 2014, 36(1):64-69.

26. Teng F, Zhu R, Zou C, Xue Y, Yang M, Song H, Liang J: Interaction between serum uric acid and triglycerides in relation to blood pressure. $J$ Hum Hypertens 2011, 25(11):686-691.

27. Alberti KG, Zimmet PZ: Definition, diagnosis and classification of diabetes mellitus and its complications. Part 1: diagnosis and classification of diabetes mellitus provisional report of a WHO consultation. Diabet Med 1998, 15(7):539-553.

28. Petersen KF, Dufour S, Befroy D, Garcia R, Shulman Gl: Impaired mitochondrial activity in the insulin-resistant offspring of patients with type 2 diabetes. N Engl J Med 2004, 350(7):664-671.

29. Michael LF, Wu Z, Cheatham RB, Puigserver P, Adelmant G, Lehman JJ, Kelly DP, Spiegelman BM: Restoration of insulin-sensitive glucose transporter (GLUT4) gene expression in muscle cells by the transcriptional coactivator PGC-1. Proc Natl Acad Sci U S A 2001, 98(7):3820-3825.

30. Gayathri SB, Radha V, Vimaleswaran KS, Mohan V: Association of the PPARGC1A gene polymorphism with diabetic nephropathy in an Asian Indian population (CURES-41). Metab Syndr Relat Disord 2010, 8(2):119-126.

31. Kunej T, Globocnik Petrovic M, Dovc P, Peterlin B, Petrovic D: A Gly482Ser polymorphism of the peroxisome proliferator-activated receptor-gamma coactivator-1 (PGC-1) gene is associated with type 2 diabetes in Caucasians. Folia Biol (Praha) 2004, 50(5):157-158.
32. Timmers $S$, Konings E, Bilet L, Houtkooper RH, van de Weijer T, Goossens GH, Hoeks J, van der Krieken S, Ryu D, Kersten S, Moonen-Kornips E, Hesselink MK, Kunz I, Schrauwen-Hinderling VB, Blaak EE, Auwerx J, Schrauwen P: Calorie restriction-like effects of 30 days of resveratrol supplementation on energy metabolism and metabolic profile in obese humans. Cell Metab 2011, 14(5):612-622.

33. Frances F, Verdú F, Portolés O, Castelló A, Sorlí JV, Guillen M, Corella D: PPARalpha L162V and PGC-1 G482S gene polymorphisms, but not PPAR-gamma P12A, are associated with alcohol consumption in a Spanish Mediterranean population. Clin Chim Acta 2008, 398(1-2):70-74.

doi:10.1186/1758-5996-6-107

Cite this article as: Wu et al:: Association and interaction analysis of PPARGC1A and serum uric acid on type 2 diabetes mellitus in Chinese Han population. Diabetology \& Metabolic Syndrome 2014 6:107.

\section{Submit your next manuscript to BioMed Central and take full advantage of:}

- Convenient online submission

- Thorough peer review

- No space constraints or color figure charges

- Immediate publication on acceptance

- Inclusion in PubMed, CAS, Scopus and Google Scholar

- Research which is freely available for redistribution

Submit your manuscript at www.biomedcentral.com/submit
C Biomed Central 